\title{
Design and Validation of a Low Cost Microscope for Diagnostics in the Developing World
}

\author{
B. Cline, ${ }^{*}$ K. Kuhlmann, ${ }^{*}$ and R. Luo** \\ *D-Rev: Design for the Other Ninety Percent, 631 Emerson Street, Palo Alto, CA 94309 \\ ** Department of Pathology, Stanford University School of Medicine, 300 Pasteur Drive, Lane \\ 235, Stanford, CA 94305
}

Introduction: Many tropical diseases, including malaria and tuberculosis, are difficult to diagnose on the basis of symptoms alone, but can be easily and accurately detected using microscope examination. Currently the expense, size, and fragility of optical microscopes impede their widespread use in the developing world. Addressing these obstacles facing microscopy in the developing world is a pressing need: over 800,000 people, primarily children in Africa, die annually of malaria and more than $1,500,000$ people die annually of tuberculosis [1][2]. The aim of this study is to design and validate a microscope for use in the developing world that combines high-resolution imaging, extreme affordability, and long-term durability.

Methods: An optical microscope with brightfield and epi-fluorescence capabilities was designed with a 10x ocular and 10x, 40x, and 100x (oil immersion) objectives. For brightfield imaging, light emitting diodes and high dispersion holographic diffuser film were used to produce 1.4 numerical aperture Köhler-equivalent illumination. For epi-fluorescence imaging, a $365 \mathrm{~nm}$ UV light emitting diode was matched with dichroic and colored glass filters. This provided illumination compatible with the broad range of stains having emissions peaks above $450 \mathrm{~nm}$. Three senior pathologists and two senior microbiologists evaluated the performance of the scope in the diagnosis of six unknown slides containing Wright-Giemsa stained peripheral blood smears for brightfield detection of malaria, Kinyoun stained sputum smears for brightfield detection of mycobacterium, and auramine stained sputum smears for fluorescent detection of mycobacterium.

Results: All five senior pathologists and microbiologists were able to make the correct diagnosis on all six unknown slides used for initial validation of the microscope (100\% concordance). By eliminating the precision-aligned optical glass elements used in traditional optical microscopes, the cost of the brightfield illumination system is reduced to less than $\$ 5$. The total estimated of production is under $\$ 150$ for brightfield illumination and under $\$ 200$ with epi-fluorescence illumination. Based on the staining techniques chosen, the estimated cost per slide is $\$ 0.07$ (comprised of $\$ 0.05$ for each glass slide and \$0.02 for staining chemicals). Use of the scope in areas without consistent access to electricity is aided by the fact that the scope can be charged by a small solar module as well as via a wall outlet. Power consumption is low: less than $1 \mathrm{~W}$ for brightfield imaging and less than $1 / 4 \mathrm{~W}$ for epi-fluorescence imaging. Due to the brightfield illumination system design, no alignment of condenser elements is required and there are few surfaces in the optical path to keep clean, an important advantage in the developing world where there is often limited technical support for microscopy. For portability, the microscope is compact (6" by 7" by 9") and lightweight at $6 \mathrm{lbs}$. As inexpensive antigen fluorochrome dyes are available for both tuberculosis (auramine rhodamine) and malaria (acridine orage), low 
magnification fluorescence screening can be faster and easier to perform while permitting overstaining for brightfield 1000x verification examination.

Conclusions: A compact, high-resolution microscope with brightfield and epi-fluorescence capabilities can be built for less than $\$ 200$. Preliminary testing suggests that the microscope enables accurate and inexpensive diagnosis of tuberculosis and malaria. Field validation of the microscope, along with testing of the diagnosis of other tropical diseases, will be conducted in conjunction with partner organizations in the developing world.

\section{References:}

[1] WHO Fact Sheet N $\mathrm{N}^{0}$. Revised January 2009.

[2] WHO Fact Sheet $\mathrm{N}^{\mathrm{o}} 104$. Revised March 2007.

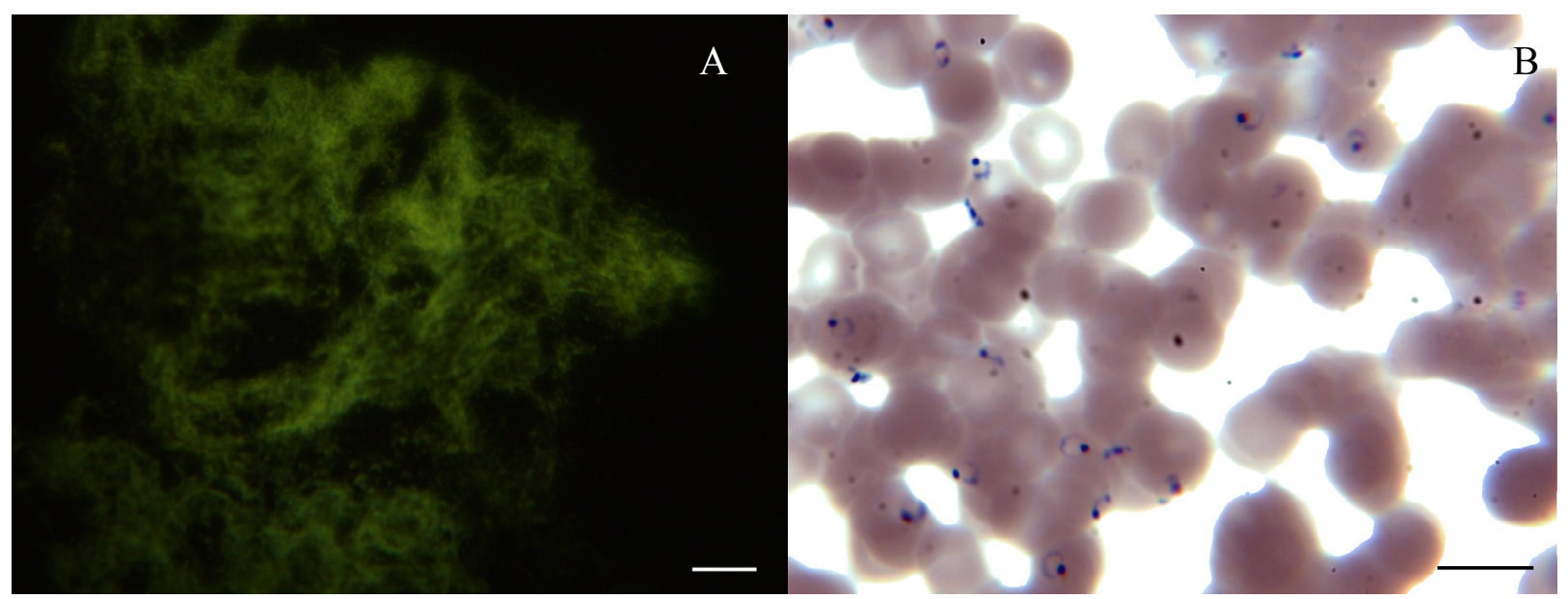

Fig. 1. Micrographs taken with the prototype low cost microscope. A. Mycobacterium (tuberculosis) imaged using epi-fluorescence illumination from an auramine stained sputum smear. B. Plasmodium falciparum (malaria) imaged using brightfield illumination from a Wright-Giemsa stained peripheral blood smear. Scale bar $=10 \mu \mathrm{m}$.

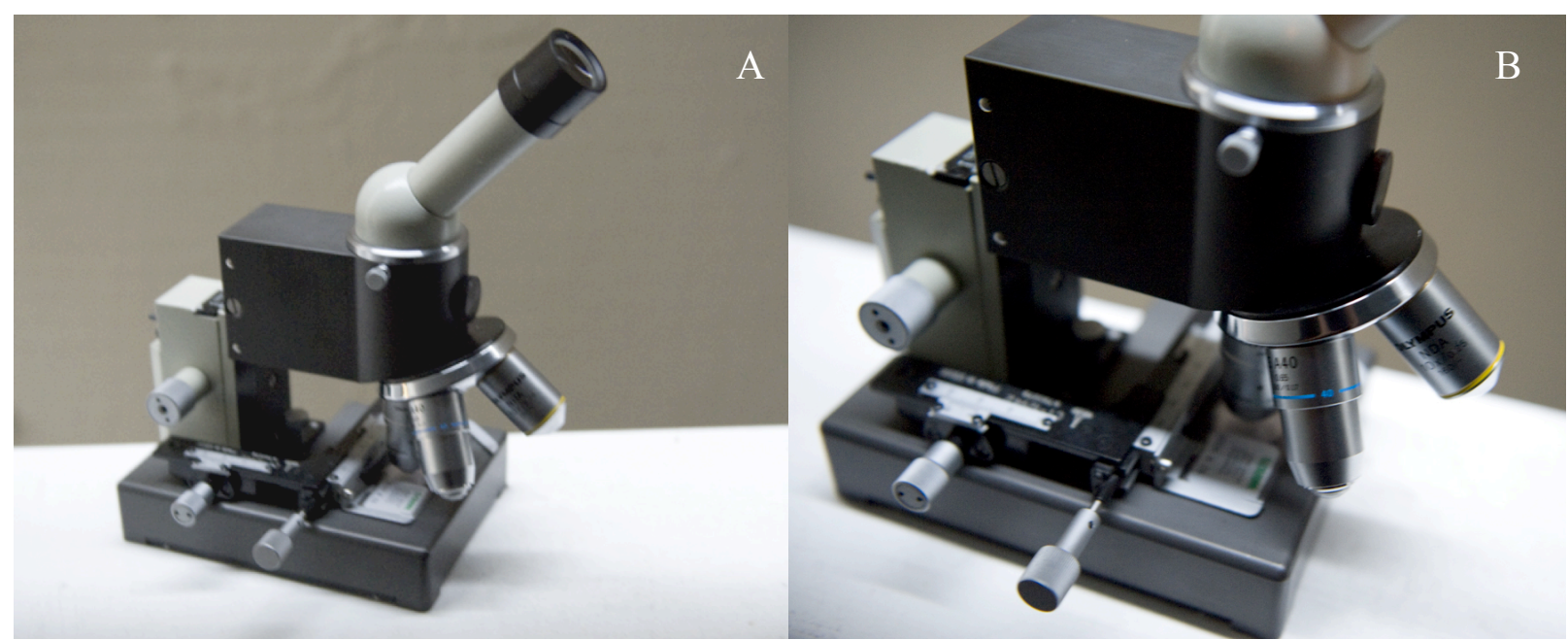

Fig. 2. Prototype of the compact, low cost microscope with both brightfield and epi-fluorescence illumination. 